\title{
THE REDUCTION OF SINGULARITIES OF PLANE CURVES BY BIRATIONAL TRANSFORMATION
}

\section{PRESIDENTIAL ADDRESS DELIVERED BEFORE THE AMERICAN MATHEMATICAL SOCIETY, DECEMBER 28, 1922}

BY G. A. BLISS

1. Introduction. In the domain of mathematics there are a number of famous theorems which have stimulated the keen interest of mathematicians over extended periods of time, and whose proofs have presented a long continued challenge to the powers of mathematical logic. To some of these, in spite of the fact that the theorems themselves remain unproved, our science is indebted for important advances.

As one illustration I may mention the well known theorem of Jordan which states that a simply closed continuous plane curve divides the plane into two and only two connected regions. This is a theorem of whose truth we are convinced at the start intuitively. But what we may for the moment call intuitive reasoning busies itself with simpler cases only, and is impatient of exceptions and refinements, unless by long study and continuous contemplation it has become a very sophisticated intuition indeed. Jordan was the first to insist that the theorem needed proof. Since his initial effort many others have attempted to give the conclusion of the theorem a substantial logical basis, and the theories of point sets and of functions of a real variable have been greatly enriched thereby. I am particularly interested to mention this theorem because it seems to me that with regard to it a satisfactory conclusion has been reached. There is doubtless still opportunity for improvements and simplifications in its proofs, but some at least of them have stood the test of examination by widely scattered experts. It is encouraging to have this evidence that not all of the mathematical questions generally recognized as most difficult are impossible to answer.

One should mention, of course, among the notable illustrations of the type of theorem which I have been discussing, Fermat's last theorem and the so-called four-color map 
theorem. The latter asserts that every map can be colored with four different colors in such a way that each country has one color only, and no two countries adjoining along a boundary have the same color. No one has constructed a map which can not be so colored, and most of us, I think, believe that the theorem is true. But so far as I know no proof of it has as yet been widely accepted, the vote usually standing one, namely the author, strongly in favor, many others doubtful, and some, who have had the temerity to examine in detail the screed in question, definitely against. This state of affairs is even more pronounced in the case of Fermat's last theorem, with the difference that one may with justice, it seems to me, feel very uncertain of the validity of the theorem itself. All of the proved evidence is in its favor, but the behavior of very large numbers, far without the range of our every-day experience, is so beyond our ken that it should not be a surprise to us if some day it were found that the theorem is not true. We are fortunate in some ways, however, that these questions remain unanswered. For every attack by experts upon them is likely to yield, as has been true notably in the past, important advances in the domains of analysis situs and the theory of numbers. The chapter of progress associated with Jordan's theorem may still be subjected to improvements and additions, but as compared with those in which the centers of interest are the four-color map theorem and Fermat's last theorem, it is relatively completed and closed.

Many of you will have inferred from the title of this address the theorem of which I wish to speak to you especially to-day. It is the one which states that by a birational transformation an algebraic curve, no matter how complicated its singularities may be, can always be transformed into another having only double points with distinct tangents. It is with some diffidence that I designate this theorem as one of the type of which I have hitherto been speaking, since among my mathematical friends there are some for whose opinions I have great respect who disagree with me as to the difficulty of its proof. Unfortunately I have not been near enough to them to find out 
with completeness what is in their minds. My purpose this afternoon will be achieved, however, if I can interest you again for the moment in some of the work of the sequence of distinguished mathematicians who have studied the theorem, and if I can make clear to those of you who have not specialized in the subject, some of the difficulties which they have sought to overcome. Up to the present time I do not know of any proof of the theorem which is sufficiently simple and unsophistocated to justify one at least of its important applications in the theory of algebraic functions to which I shall presently refer again.

2. The Two Transformation Theorems. In the statement of the theorem mentioned in $\S 1$, reference is made to the notion of a birational transformation. There are two types of birational transformations, one of which is well illustrated by the very simple but important example $\xi=x, \eta=x / y$ relating the points of the $x y$-plane to those of the $\xi \eta$-plane. If one prefers homogeneous coordinates this transformation may be written in the form $\rho \xi_{1}=x_{2} x_{3}, \rho \xi_{2}=x_{3} x_{1}, \rho \xi_{3}=x_{1} x_{2}$. It establishes a one-to-one correspondence between the points of the two planes with the exception of those on certain loci, in this case the coordinate axes, and is a special example of the general class of birational transformations which establish such correspondences between two planes and which are called Cremona transformations. Incidentally the transformation transforms every not too special algebraic curve $f(x, y)=0$ of the $x y$-plane into a similar curve $\varphi(\xi, \eta)=0$ of the $\xi \eta$-plane and establishes a one-to-one birational correspondence between their points. It is also possible, however, to have a one-to-one correspondence between the points of two curves $f(x, y)=0$, $\varphi(\xi, \eta)=0$, with coordinates $(x, y)$ and $(\xi, \eta)$ of corresponding points rationally expressible in terms of each other, but such that the correspondence is not a part of any Cremona transformation between the planes of the two curves such as has been described above. Such a transformation has been called a birational transformation relating the two curves alone. A simple example of such a transformation is defined by the 
equations $\xi=x^{2}, \eta=y$ when applied to a curve not symmetric with respect to the $y$-axis.

Before proceeding to the discussion of the various proofs which have been made of the theorem I should like to say that there are really two closely related theorems which have been considered in this connection. To understand them we should remember that an "ordinary" singular point of a curve is one through which pass a finite number of branches whose tangents are distinct. For such a point $(x, y)=(a, b)$ in the finite part of the plane these branches will be determined analytically by convergent series of the form

$$
x=a+\alpha t+\cdots, \quad y=b+\beta t+\cdots,
$$

in which the coefficients $\alpha$ and $\beta$ are not both zero, and the different branches through $(a, b)$ will have distinct ratios $\alpha: \beta$. The two theorems mentioned are then as follows.

Theorem I. Every irreducible algebraic plane curve can be transformed into another which has only ordinary singular points by a Cremona transformation of the planes of the two curves.

THeOREM II. Every irreducible algebraic plane curve can be transformed into another which has only ordinary double points by a transformation which is birational between the two curves.

Theorem II is the one of which I have already spoken, and I may say here that there are two types of proofs of it. For one of them the conclusion of Theorem I is assumed, and a further birational transformation is then determined which will replace ordinary multiple points of multiplicity higher than two by ordinary double points. The other type of proof is more direct, presenting at once a birational transformation producing ordinary double points only, without the use of Theorem I.

In the theory of algebraic functions these two theorems have an important application. The genus of an algebraic curve, and the more important properties of the Abelian integrals and the field of rational functions associated with the curve, are invariant under birational transformations. The 
presentation of the theory, from the standpoint of the so-called transcendental methods inaugurated by Riemann and developed and amplified by many other writers, is greatly simplified if one can presuppose that the curve under consideration has only ordinary singular points. Some further advantage in simplicity would be gained if only ordinary double points were present, but, as one of my mathematical colleagues has aptly said, this would be in the nature of a luxury rather than a necessity. It is significant, however, that most lecturers and writers who approach the subject from the standpoint of Riemann are content to start with the result of Theorem I, which they probably would not do if they found that Theorem II were provable in relatively as simple and elementary a fashion. Some of the other theories of algebraic functions which do not presuppose any special properties of the singular points of the basal curve seem to me highly interesting" and desirable, notably that of Dedekind and Weber as amplified and presented by Hensel and Landsberg.* Theoretically this is certainly a very elegant and satisfying method of approach.

In speaking a few minutes ago of the correspondence established between two algebraic curves by a birational transformation, I used locution of which I very much disapprove, but which at that moment could not very well be explained. The elements of an algebraic curve which are set into one-to-one correspondence with those of another curve by a birational transformation are not the points of the curve, but the so-called branches or cycles. Through each finite point of the curve there passes one of these branches, if the point is simple, but possibly more than one if the point is multiple, and there are besides branches belonging to the so-called points at infinity. Analytically each branch is determined in non-homogeneous coordinates by equations of the form $x=P(t), y=Q(t)$, where $P(t)$ and $Q(t)$ are convergent power series having usually only terms with positive powers of $t$, but for a finite number of branches having also a finite number of terms with negative exponents. If homogeneous coordi-

* Theorie der algebraischen Funktionen einer Variabeln, 1902. 
nates are used the cycle or branch can always be represented by series having only positive powers of $t$. Weierstrass especially emphasized the point of view that a curve may well be thought of as the totality of the branches which satisfy its equation, and I think that failure to appreciate his contention has in some cases caused confusion in the proofs of the theorems of which I have been speaking. Birational transformations do not in general transform curves point for point into each other, but they do establish a unique correspondence between the branches of the two curves.

3. Geometric Interpretations. The most useful geometric picture of an algebraic equation $f(x, y)=0$ which we have is its so-called Riemann surface. The effectiveness of the picture is due to the fact that there is a one-to-one continuous correspondence between the points on the surface and the branches which satisfy the equation. Furthermore the character of the cycle of sheets of which each point of the surface is a vertex indicates important properties of the corresponding analytic branch. Besides the Riemann surface, however, there are two other geometric representations of an algebraic curve which have especial importance in connection with the transformation theorems stated above, but each of which is open to the objection that it visualizes for us only the real branches of the algebraic curve in question.

For the first of these, consider the pencils of lines through two points $A$ and $B$ selected arbitrarily in the plane. In the pencil through $A$, for example, we may regard each ray as designated by the value $x$ of its anharmonic ratio with three fixed rays through $A$ chosen in advance; and the rays through $B$ are similarly determined by numbers $y$. If we now associate with each pair of values $(x, y)$ satisfying an algebraic equation $f(x, y)=0$ the point of intersection of the corresponding $x$ and $y$-rays through $A$ and $B$, we obtain a curve representing geometrically the real branches of our equation. In order to give equal consideration to all the rays through $A$ and $B$ without using infinite values for the coordinates we may introduce the homogeneous variables $x_{1}, x_{2}$, and $y_{1}, y_{2}$ defined by the equations $x=x_{1} / x_{2}, y=y_{1} / y_{2}$. 
The other geometric representation of the equation $f(x, y)$ $=0$ is so familiar that I need not speak of it in detail. If we replace $x$ and $y$ by homogeneous coordinates $x_{1}, x_{2}, x_{3}$ defined in the usual way by the equations $x=x_{1} / x_{3}, y=x_{2} / x_{3}$, then the equation $f(x, y)=0$ determines a curve in the projective plane as soon as a coordinate triangle and unit point have been assigned.

For the sake of convenience let us agree to call the former of these two geometric interpretations of the equation $f(x, y)$ $=0$ the function-theoretic one, and the latter simply the geometric interpretation. The reason for this nomenclature is that the former is closely allied to the theory of algebraic functions, while the latter is that of ordinary plane projective geometry. The function-theoretic interpretation is the more convenient one for representing properties of curves which are invariant under transformations of the form

$$
x=\frac{a_{1} \xi+a_{2}}{a_{3} \xi+a_{4}}, \quad y=\frac{b_{1} \eta+b_{2}}{b_{3} \eta+b_{4}},
$$

while the geometric interpretation has similar advantages for transformations

$$
x=\frac{a_{1} \xi+b_{1} \eta+c_{1}}{a_{3} \xi+b_{3} \eta+c_{3}}, \quad y=\frac{a_{2} \xi+b_{2} \eta+c_{2}}{a_{3} \xi+b_{3} \eta+c_{3}} .
$$

We say in either case that a point of the curve $f(x, y)=0$ with finite or infinite coordinates is an ordinary double point if it has this character after an appropriate transformation (1) or (2) into a non-special point with finite coordinates. The points on the line $A B$ in the function-theoretic representation are special points.

It is perhaps more satisfactory to define analytically what is meant by a curve with ordinary double points only. In the function-theoretic case the equations of a branch can always be taken in the form

$$
\begin{gathered}
x_{i}=a_{i}+\alpha_{i} t+\cdots, \quad y_{i}=b_{i}+\beta_{i} t+\cdots, \quad(i=1,2), \\
\left(a_{1}, a_{2}\right) \neq(0,0), \quad\left(b_{1}, b_{2}\right) \neq(0,0),
\end{gathered}
$$

and the branch is said to be linear if the two determinants

$$
a_{1} \alpha_{2}-a_{2} \alpha_{1}, \quad b_{1} \beta_{2}-b_{2} \beta_{1}
$$


do not both vanish. A second branch with primed coefficients has the same center if

$$
a_{i}^{\prime}=k a_{i}, \quad b_{i}^{\prime}=l b_{i}, \quad(i=1,2) ;
$$

and two branches with the same center are said to have distinct tangents if the expression

$$
\left(a_{1} \alpha_{2}{ }^{\prime}-a_{2} \alpha_{1}{ }^{\prime}\right)\left(b_{1}{ }^{\prime} \beta_{2}-b_{2}{ }^{\prime} \beta_{1}\right)-\left(a_{1}{ }^{\prime} \alpha_{2}-a_{2}{ }^{\prime} \alpha_{1}\right)\left(b_{1}{\beta_{2}}^{\prime}-b_{2} \beta_{1}{ }^{\prime}\right)
$$

is not zero.

In the geometric case a branch has equations

$$
x_{i}=a_{i}+\alpha_{i} t+\cdots, \quad(i=1,2,3),\left(a_{1}, a_{2}, a_{3}\right) \neq(0,0,0),
$$

and is linear if the determinants $a_{i} \alpha_{k}-a_{k} \alpha_{i}(i \neq k)$ are not all zero. A second branch with primed coefficients has the same center if $a_{k}{ }^{\prime}=\rho a_{k}(k=1,2,3)$; and two branches with the same center have distinct tangents if the determinant $\left(a_{1} \alpha_{2} \alpha_{3}{ }^{\prime}\right)$ is different from zero. For branches with finite centers $(x, y)=(a, b)$ these definitions of branches with distinct tangents reduce to those of the usual cartesian analytic geometry. A curve with no singularities except ordinary double points is in either interpretation one such that all of its branches are linear, such that none of its points is a center of more than two of its branches, and such that every pair of its branches having a common center has distinct tangents. These properties are invariant in the function-theoretic and geometric cases under the respective transformations (1) and (2).

Now it happens that the branches which satisfy an equation $f(x, y)=0$ may determine a curve with ordinary double points only in one of these interpretations, while more complicated singularities are present in the other. This is due to the fact that we have added in two different ways points with infinite coordinates to the totality of finite points $(x, y)$ in the plane in order to obtain the two interpretations. If, for example, the line $x=\infty$ through $A$ is intersected by $n$ distinct branches in the function-theoretic case, then the curve for the geometric interpretation of the equation will have an ordinary singular point with $n$ branches at the intersection of the $x$-axis with the line at infinity. Similarly if the line at 
infinity in the projective plane intersects the geometric curve in $n$ points distinct from each other and from the $x$-and $y$-axes, then the function-theoretic curve will have an ordinary singular point with $n$ branches through the intersection of the lines $x=\infty$ and $y=\infty$.

We could readily analyze this correspondence in greater detail, but the essential thing to remark is that there is really a pair of Theorems II which are not equivalent. The distinction between these theorems has not, in my opinion, been sufficiently emphasized in the literature, though proofs applying to both have been presented. At the present time the function-theoretic theorem appears to me somewhat easier to handle, though neither of them is simple.

4. Noether's Theorem. Those of you who have not been specialists in this domain will perhaps be interested to know something of the origins of these theorems. Theorem I is, with what seems to be universal agreement, ascribed to Noether. In $1871^{*}$ he stated the theorem and indicated the proof which has since been modified and elaborated in one way or another by many writers. A difficulty in the proof not explained in this original paper can be removed by methods used in the same year by Hamburger $\uparrow$ in his study of the Puiseaux expansions at a singular point. Noether $\ddagger$ himself in 1876 treated the question more elaborately in a paper devoted to the analysis of the singular points of an algebraic curve. Bertini $\S$ was dissatisfied with the methods used by Noether, and in 1888 gave a direct proof of the theorem which he characterizes as simple and rigorous. The proof with which $I$ am most familiar is that given by Picard $\|$ and ascribed by him to Simart. The geometers would be perhaps most interested in those of Severi $\uparrow$

\footnotetext{
* Göttinger Nachrichten, 1871, page 267.

$\dagger$ Zieitschrift für Mathematik und Physik, vol. 16 (1871), p. 461.

$\ddagger$ Mathematische Annalen, vol. 9 (1876), p. 166, especially $\S 5$.

§ Lombardo Rendiconti, (2), vol. 21 (1888), page 326. In commenting on Noether's paper he says "le considerazioni ( $\$ 5$ ) lasciano forse a desiderare maggiore semplicita e chiarezza," and he says that his intention in his own paper is to prove Noether's theorem "semplice e rigorosa."

|| Traité d'Analyse, vol. 2, 1st ed., 1893, page 360; 2d ed., 1905, p. 404.

I Lezioni di Geometria Algebrica, 1908, p. 61.
} 
and Enriques,* but there are many others. $\dagger$ The proofs are all modifications of Noether's original one, and there is no question with regard to the validity of the theorem. I may add that Halphen $\ddagger$ gave two methods of transforming a curve into one with ordinary singular points only by birational transformations which are not Cremona transformations.

The method of the proof is to place the origin of coordinates at one of the singular points of the curve, say one of order $k$, and then to apply the simple quadratic transformation $\xi=x$, $\eta=x / y$ of which I have spoken above. In the new curve the character of the singular points not at the origin remains the same as before, but the singularity of order $k$ at the origin is exploded by the transformation, and when one examines the pieces remaining two new ordinary singular points are found at corners of the coordinate triangle, plus one or more other fragmentary singular points on one side of this triangle the sum of whose orders is $k$. The difficulty which I have men tioned above and which was not originally explained by Noether lies in the possibility that there may be only one such fragment of the same order as the original singularity, in which case the situation might easily be as bad after as before the transformation. It is provable, however, that after a finite number of repetitions of the transformation the number of the fragments will surely be greater than one, and their orders each less than $k$. By a continuation of the process, therefore, one can finally replace the singular point originally placed at the origin, and indeed all of the singular points, by ordinary singular points only. It is not surprising that there have been so few variations from the method of proof originally

* Lezioni sulla Teoria Geometrica delle Equazioni e delle Funzioni Algebriche, vol. 2,1918 , p. 417.

$\dagger$ See EnCYClopäDIE, III C 4, p. 363; Pascal, Repertorium der Höheren Mathematik, vol. 2, 2d ed., p. 291; Brill und Noether, Die Entwicklung der Theorie der algebraischen Functionen, JAHRESBERICHT DER VEREINIGUNG, vol. 3 (1892-3), p. 369. Interesting references which do not seem to be listed in these places are Jordan, Cours d'Analyse, vol. 1, 2d ed., 1893, p. 588; and Clebsch-Lindemann, Vorlesungen über Geometrie, vol. 1, 1876, p. 491 .

$\ddagger$ Comptes Rendus, vol. 80 (1875), p. 638, and Oeuvres, vol. 1, p. 358; Journal de Mathématiques, (3), vol. 2 (1876), p. 87. 
proposed by Noether, if one remembers the theorem that every Cremona transformation is decomposable into a succession of projective transformations and simple quadratic transformations of the type we have been considering.

5. The Kronecker Group of Proofs of Theorem II. With regard to Theorem II, it seems to me that the story is quite a different one. The origin of the theorem is less easy to determine, and no proof of it has been adopted with universal or even with widespread approval. During a somewhat cursory examination of the literature over a year ago I found so many doubtful comments by authors themselves upon proofs which had preceded theirs, and so many questions of my own which it seemed difficult to answer, that I resolved to try by a closer personal examination to convince myself of the merits of some of the published demonstrations of the theorem. It is of the impressions gained from this study that I wish to speak to you now. After we have looked for a moment at the various types of proofs which have been given it will perhaps be easier to describe intelligibly the sources from which the theorem seems to have been developed.

The proofs fall into four main groups,* and I shall speak first of that which originated with Kronecker because it is the one in which I have myself been primarily interested. In 1881 Kronecker $\dagger$ published a paper in which he discusses the factorization of the discriminant of an algebraic function, and which he had presented to the Berlin Academy many years before, in 1862. It is a very well known paper, for many reasons one of the most suggestive and interesting which $\mathrm{I}$ have ever read. In it he shows that the discriminant of an algebraic function $y$ of $x$, defined by an algebraic equation $f(x, y)=0$, has always the form $D=R^{2} \Delta$, where $R$ and $\Delta$ are two polynomials in the variable $x$. If the equation $f(x, y)=0$ is subjected to a birational transformation of the form

$$
\xi=x, \quad \eta=r(x, y)
$$

* For the literature see Brill and Noether, loc. cit., pp. 369 ff.; Wirtinger, ENCYCLOPÄDIE, II B 2, p. 127; Berzolari, ibid., III C 4, pp. 362 ff.; and in Pascal's Repertorium, loc. cit.

$\dagger$ JoURNAL FÜR MATHEMATIK, vol. 91 (1881), p. 301. 
upon the dependent variable alone, then the resulting equation $\varphi(x, \eta)=0$ will have a discriminant $D_{1}=R_{1}{ }^{2} \Delta$ in which $R_{1}$ is in general different from $R$, while the factor $\Delta$ remains unchanged. A principal purpose of the paper is to determine the birational transformation in such a way that the roots of $R_{1}$ are distinct from each other and from those of $\Delta$. If this has been accomplished, then it is provable that the curve $\varphi(x, \eta)=0$ can have no singularities in the finite part of the $x \eta$-plane except ordinary double points. This last step I do not find explicitly explained in Kronecker's paper, though it is clear from other sources that he and his followers were well aware of the geometric significance of his transformation of the discriminant.

Kronecker states that the results of the paper. of which I have been speaking were in part known to him as early as 1857 , and that he used them repeatedly in his lectures for many years before the paper itself was published. His transformation of the discriminant was adopted by Weierstrass in lectures on the theory of algebraic functions in 1869. I am acquainted with Weierstrass' treatment of the question only through a brief outline by Brill and Noether* and a more detailed paper based upon this outline published by Thomé in 1903. $\dagger$ The papers of Kronecker and Thomé are both very clear and convincing, but neither of them affords a complete proof of either the function-theoretic or the geometric Theorem II because after their transformations there may still be complicated singularities at infinity, though only ordinary double points in the finite part of the plane.

In order to secure his birational transformation simplifying the discriminant, Kronecker made use of the rational functions $\eta(x, y)$ on the Riemann surface of the curve $f(x, y)=0$ which have no poles on the finite part of the surface, the so-called integral algebraic functions in the field of rational functions associated with the curve. A general formula for such a function is

(3) $\quad \eta(x, y)=u_{1}(x) \eta_{1}(x, y)+\cdots+u_{n}(x) \eta_{n}(x, y)$,

* Loc. cit., pp. 375-6.

† JoURNAL FÜr MAThematik, vol. 126 (1903), p. 52. 
where $\eta_{1}, \cdots, \eta_{n}$ is a suitably selected set of linearly independent functions of this sort, and the coefficients $u_{1}, \cdots, u_{n}$ are arbitrary polynomials in $x$. If these coefficients $u_{k}$ are suitably specialized, then $x$ and $\eta$ will satisfy an algebraic equation $\varphi(x, \eta)=0$ birationally related to $f(x, y)=0$ and with a discriminant of the desired simplicity.

In 1902 Hensel and Landsberg* extended this reasoning of Kronecker. The functions $\eta(x, y)$ which Kronecker uses are restricted to have minimum orders zero at the finite points of the Riemann surface of $f(x, y)=0$, but are unrestricted at the infinite points. A formula like (3) still holds, however, if the functions $\eta(x, y)$ have the properties just mentioned with the exception that minimum orders different from zero, either positive or negative, are admitted at a limited number of finite points of the Riemann surface. If now the number of poles allowed for $\eta(x, y)$ is sufficiently large, and if the coefficients $u_{1}, \cdots, u_{n}$ are again suitably specialized, the function $\eta(x, y)$ will not only satisfy an equation $\varphi(x, \eta)=0$ birationally related to $f(x, y)=0$, and have a discriminant of the form attained by Kronecker, but it will also have finite distinct values at the infinite points of the Riemann surface. This brings us to the function-theoretic Theorem II provided that the poles allowed for $\eta(x, y)$ are of order one and over distinct values of $x$. For if on the Riemann surface of $\varphi(x, \eta)$ $=0$, which is the same as that for $f(x, y)=0$, the function $\eta(x, y)$ has distinct finite values at the value $x=\infty$ and simple poles over distinct finite values of $x$, then the infinite branches of $\varphi(x, \eta)=0$ give rise to no multiple points in the functiontheoretic interpretation of the equation. If furthermore the discriminant of $\eta(x, y)$ has the simple form attained by Kronecker, then the curve $\varphi(x, \eta)=0$ can have no singularities at finite points $(x, \eta)$ except ordinary double points.

The discussion of the separation of the roots of the discriminant given by Hensel and Landsberg seems to me at one point incomplete $\dagger$ without the addition of some

* Theorie der algebraischen Funktionen einer Variabeln, pp. 402-9.

$\dagger$ Loc. cit., the paragraph on pages $407-8$. 
details from Kronecker's original memoir. Their final statement of the theorem does not distinguish between the functiontheoretic and geometric cases upon which I have insisted above, and it is followed by the assertion without a reference that the theorem was first proved by Kronecker. So far as I can discover from published papers, this last statement is not quite just to themselves. The extension of Kronecker's proof which enables one to secure proper behavior of the transformation at infinite as well as finite points of the Riemann surface is apparently their own, and it is a much more difficult extension than is indicated by my brief outline of their argument.

I have been interested to attempt to prove the geometric Theorem II also by Kronecker's method, and have found a proof which seems to me satisfactory. If an algebraic equation $\varphi(\xi, \eta)=0$ of degree $q$ defines $q$ expansions for $\eta$ at $\xi=\infty$ of the form

$\eta=\alpha_{k} \xi+\beta_{k}+\gamma_{k} \frac{1}{\xi}+\cdots, \quad\left(k=1, \cdots, q ; \alpha_{k} \neq \alpha_{l}\right.$ for $\left.k \neq l\right)$,

and if the discriminant for $\eta$ has the simplified form attained by Kronecker, then it is provable that in the projective plane the corresponding curve meets the line at infinity in distinct points and has no singularities in the finite part of the plane except ordinary double points. To obtain such a curve birationally related to $f(x, y)=0$ one can start by selecting $q>2 p+2$ ordinary points on the Riemann surface of $f(x, y)=0$ over distinct values of $x, p$ being the genus of the surface. The functions $\eta(x, y)$ which have no singularities except possibly simple poles at these points constitute a linear family of the form

(4) $\eta(x, y)=c_{1} \eta_{1}(x, y)+\cdots+c_{\nu} \eta_{\nu}(x, y), \quad(\nu=q-p+1)$, the coefficients $c_{k}$ being constants. A suitable special choice of these coefficients can easily be made so that the resulting function $\xi(x, y)$ has surely a simple pole at each of the $q$ points. With more difficulty a second special function $\eta(x, y)$ of the family can then be chosen which with $\xi$ satisfies an equation $\varphi(\xi, \eta)=0$ birationally related to $f(x, y)=0$ and which has the further properties described above. 
Here, then, based upon the methods of Kronecker, are proofs of both the function-theoretic and geometric Theorems II. They are what I should call sophisticated proofs, since they make use of properties of rational functions with prescribed poles on the Riemann surface of $f(x, y)=0$, which are well known and well established but not elementary. These properties are proved in the theory of algebraic functions as presented by Hensel and Landsberg without restrictive assumptions upon the singularities of $f(x, y)=0$. If the socalled transcendental method of Riemann is adopted, however, it is usually presupposed in proving them that the singularities of the curve $f(x, y)=0$ are ordinary multiple points only, or, in other words, that the result of Theorem I has been attained by transformation. An easily derived property which I have found for the linear families (4) aids very much in simplifying the proofs of both the function-theoretic and geometric interpretations of Theorem II.

6. The Halphen Group of Proofs of Theorem II. There is a second very interesting series of discussions of Theorem II which seems to have been inaugurated by Halphen in $1884 .^{*}$ So far as I have been able to discover this paper of Halphen's contains the first explicit statement and proof of Theorem II which has been published. In 1893 Picard $\dagger$ reproduced Halphen's argument. At its conclusion, in commenting on a particular step in the proof, he says that the point is not to be doubted, but that he does not see quite the means of establishing it so as to avoid every objection. The reasoning by continuity which he has just been using makes the accuracy of the conclusion seem to him more than probable. In a footnote he adds that Halphen arrives at the theorem without mentioning the difficulty.

This comment of Picard seems to have instigated a series of attacks on the theorem which continued for more than a

* Étude sur les points singuliers des courbes planes algébriques, appendix to Salmon's Traité de Géométrie Analytique (Courbes Planes), translated by O. Chemin, edition of 1903, pp. 627-31.

$\dagger$ Traité d'Analyse, vol. 2, 1893, p. 364. 
decade. In the same year, 1893, Simart* modified Halphen's proof as presented by Picard and remarks that the theorem, though affirmed by many authors, has never to his knowledge been demonstrated rigorously, and he offers, therefore, a demonstration which he believes to be secure against every objection. Only a little later in the same year Poincaré $\dagger$ proposed a geometric proof of a different sort, of which he says that the two principal steps will possibly seem almost evident. Yet there will perhaps be some interest, he continues, in having a demonstration which is secure against every objection. In 1895 Appell and Goursat $\ddagger$ refer in a footnote to the proofs of Simart and Poincaré, but rely for their establishment of the theorem upon another very interesting transformation previously used by Halphen $\S$ to secure a curve with ordinary singular points only. In 1896 Vessiot /i states that many demonstrations of the theorem have been given, but that the modification of Appell and Goursat's proof which he is about to explain will not be without interest on account of its simplicity and complete rigor. In a footnote 9 he says that if one accepts as evident the steps $(e)$ and $(f)$ of his demonstration, as Appell and Goursat have done at the analogous points of their proof, one may arrive at his conclusion more rapidly. But the explanations which he gives seem to him indispensable if one desires to establish these two points beyond all doubt.

It seems to me that these comments express considerable dissatisfaction and uncertainty with regard to the proofs of Theorem II which were at that time under discussion. The transformations suggested by Halphen are most interesting and plausible, and one would expect, as Picard implies, that their effectiveness can be conclusively established. I do not feel convinced as yet that this has been done. Simart's paper is so concise that I have so far been unable to verify all the

* Comptes Rendus, vol. 116 (1893), p. 1047.

$\dagger$ Comptes Rendus, vol. 117 (1893), p. 18.

¥Théorie des Fonctions Algébriques, 1895, p. 282.

§ Journal de Mathématiques, loc. cit.

|| AnNales de Toulouse, vol. 10 (1896), p. D1.

T) Page D6. 
steps of his argument to my own satisfaction. Vessiot uses a combination of projective transformations with the simple transformation

$$
\xi=x, \quad \eta=\frac{d y}{d x}=-\frac{f_{x}}{f_{y}} .
$$

One cannot in general by a preliminary projective transformation prevent this function $\eta$ from having poles in the finite part of the Riemann surface of $f(x, y)=0$. Each of these poles provides a branch which in the projective $x \eta$-plane passes through the intersection of the $\eta$-axis and the line at infinity. A singular point is therefore introduced at infinity on the projective curve which cannot be made to disappear by a subsequent projective transformation. It does not seem possible, therefore, to prove the geometric Theorem II by this method. On the other hand I am inclined to believe that Vessiot's method may afford a relatively simple proof of the function-theoretic Theorem II. By a projective transformation one should be able to bring it about that $\eta=d y / d x$ has distinct finite values at the infinite points of the Riemann surface, and at most simple poles over distinct values of $x$ in the finite parts of the surface. These properties insure the existence of simple points only at the intersections of the function-theoretic curve with the rays $x=\infty$ and $y=\infty$ through the points $A$ and $B$. The methods of Vessiot could then be used to secure at most ordinary double points at finite points $(x, y)$. Some of the steps are, however, analytically delicate.

7. The Bertini Group of Proofs of Theorem II. A third group of proofs, this time of the geometric Theorem II, is due to Bertini.* In 1891 he was apparently unaware of Halphen's paper of 1884 , for he states that in many works on geometry Theorem II is applied, but that as far as he knows no one has given an explicit demonstration. He proposes, therefore, one which appears to him to be simple and rigorous. It seems likely that the form which he devised was suggested by some studies of birational transformations of curves published by Clebsch and Lindemann in 1876. $\dagger$ In 1894 Bertini republished

* Rivista di Matematica, vol. 1 (1891), p. 22.

$\dagger$ Vorlesungen über Geometrie, 1876, pp. $661 \mathrm{ff}$. 
his proof in the Mathematische Annalen, ${ }^{*}$ and in a footnote explained that a geometric difficulty which Poincaré had avoided somewhat circuitously in 1893 had already been discussed by himself. Klein added a second footnote in which he suggested an interesting geometric transformation, equivalent to Bertini's, which he says had been communicated to him orally by Clebsch in 1869 . In 1905 , in the second edition of the second volume of his Traité d'Analyse, $\uparrow$ Picard adopts Bertini's proof with slight modifications only. In 1906 Walker $\ddagger$ remodeled Bertini's method in detail in accordance with Klein's geometric suggestion. In Walker's introduction he refers to the proofs listed in this and the preceding groups, and remarks that they are written in very concise style and leave a great many minor points to the reader. In 1907 W. H. and G. Ch. Young $\S$ described a new condition on Bertini's transformation which must be added in order to make it entirely satisfactory. They were evidently unacquainted with Walker's thesis, in which this objection had been answered in a different way but had not been pointed out explicitly.

The great advantage of the form of Bertini's proof adopted by Walker lies in the fact that the various steps of the proof have geometric interpretations in ordinary spaces of two and three dimensions. It is consequently easier by this method to classify the properties which must be imposed upon Bertini's transformation, and to make sure that all of them have been suitably secured. The proof is in outline as follows. Through six points of a plane $\pi$, not on a conic and no three collinear, there pass four linearly independent cubic curves $f_{i}\left(x_{1}, x_{2}, x_{3}\right),(i=1, \cdots, 4)$. The equations

$$
\rho y_{i}=f_{i}\left(x_{1}, x_{2}, x_{3}\right), \quad(i=1, \cdots, 4)
$$

define a cubic surface $F$ in the three-dimensional $y$-space, and the points of $F$ are in one-to-one correspondence with those of the plane $\pi$ with the exception that six of the straight lines

\footnotetext{
* Vol. 44 (1894), p. 158.

$\dagger$ Page 408.

$\ddagger$ On the resolution of higher singularities of algebraic curves into ordinary nodes, Dissertation, Chicago, 1906.

§ Torino Atti, vol. 42 (1907), pp. 82-6.
} 
on the surface correspond to the six base points. For each of these lines there is, however, a one-to-one correspondence between its points and the rays through the corresponding base point. Let $K$ be an irreducible algebraic curve in the $x$-plane with ordinary singular points only, and let the base points of the transformation just described be selected so that one of them is at a singularity $A$ of $K$ but the others not on $K$. Then the curve $K$ is transformed by equations (5) into a new curve $K^{\prime}$ on the surface $F$, and $K^{\prime}$ has singularities similar to those of $K$ with the exception that one of them, namely $A$, is dispersed into ordinary points. If a suitable center of projection $O$ is now taken on the surface $F$, the curve $K^{\prime}$ can be projected into a plane curve $K^{\prime \prime}$ which has possibly new ordinary double points in place of $A$, but whose other singularities are the same in number and in type as those of $K$. By a succession of such transformations all of the singular points of $K$ may therefore be replaced by ordinary double points.

The difficult part of the demonstration is the proof that it is possible to choose properly the center of projection $O$. If $O$ is selected on $F$ but distinct from $K^{\prime}$ and the straight lines of $F$, and distinct from the cones generated by $K^{\prime}$ at its own singular points, then the projection of $K^{\prime}$ from $O$ defines a curve $K^{\prime \prime}$ in one-to-one correspondence with $K^{\prime}$ except for a finite number of double points of $K^{\prime \prime}$ where the correspondence is one-to-two. The choice of $O$ in this manner on the cubic surface $F$ effectively prevents any ray through $O$ from being a trisecant of $K^{\prime}$. If $O$ is not on the developable of the tangents to $K^{\prime}$, then $K^{\prime \prime}$ will have no cusps. If $O$ is not on the tangent planes to $F$ at the multiple points of $K^{\prime}$, and not on the cones generated by $K^{\prime}$ at its own singular points, then each multiple point of $K^{\prime}$ will project into an ordinary multiple point of the same character for $K^{\prime \prime}$. Finally if $O$ is distinct from the ruled surface whose generators are lines joining points of $K^{\prime}$ with coplanar tangents, then the only double points introduced for $K^{\prime \prime}$ will be ordinary double points. In order to make the proof complete it must be shown that each of the loci just mentioned exists and can be avoided in the 
selection of the point $O$. Walker has not only done this but he has also discussed in detail the properties of the cubic transformation from the plane $\pi$ to the surface $F$. His thesis fills all told about fifty pages, a decided contrast to Bertini's original outline of slightly more than one page. I have been satisfied, by Walker's reasoning or by arguments of my own which seemed to me more convenient, that all of his steps are well justified. It would be highly desirable to have the proof shortened and simplified without sacrificing clearness or accuracy, and I am inclined to think that it could be done.

8. Other Geometric Proofs of Theorem II. There is a fourth and final principal group of proofs of Theorem II, more geometric in character, with which I am relatively much less familiar.* The plan underlying most of them is to transform the given plane algebraic curve into a twisted curve in a higher space devoid of singularities, and then to project this non-singular curve upon a two-dimensional plane in such a way that only ordinary double points remain. Intuitively the possibility of these two steps, as Poincare says, does seem almost evident, and it is perhaps for this reason that in most of the geometric proofs the final projection is hardly mentioned. It seems to me, however, that this projection is a really difficult part of the proof. Poincarés proof, for example, is very condensed, but all of his steps can be verified without serious difficulty up to his discussion of the projection of the non-singular curve in higher space into a non-singular curve in three-space. At this point his reasoning seems to me much too concise, and even questionable. In Walker's form of Bertini's proof the projection of the space curve $K^{\prime}$ on the surface $F$ into a plane curve $K^{\prime \prime}$ is the most difficult step. My doubts about the ease of making the projection have been strengthened by

* Veronese, Mathematische Annalen, vol. 19 (1881), p. 213; Poincaré, loc. cit.; del Pezzo, Napoli Rendiconti, (2), vol. 7 (1893), p. 15; Vessiot, Bulletin de la Sociéte Mathématique de France, vol. 22 (1894), p. 208; Pieri, Rivista di Matematica, vol. 4 (1894), p. 40; Segre, Annali di Matematica, (2), vol. 25 (1897), p. 43; Severi, Lezioni di Geometria Algebrica, 1908, p. 172; Del Re, Modena Memorie, (2), 10 (1894), p. 447, footnote; Jamet, Nouvelles Annales, (3), vol. 19 (1900), p. 506. 
some further interesting evidence. After I had made the proof of the geometric Theorem II, following Kronecker, I read Severi's geometric proof and was surprised to find a close analogy between my own argument and his. This was a result of the relationship between the theory of rational functions of $x, y$ with prescribed poles on the Riemann surface of $f(x, y)=0$ and the geometric theory of the groups of points intersected on the curve $f(x, y)=0$ by linear families of curves. It is these linear families which Severi uses to get his transformation from the plane curve to a non-singular curve in higher space. But Severi relegates the question of the projection of his space curve upon a plane, so as to leave only ordinary double points, to a short footnote containing only some remarks on how to avoid trisecants, whereas in the Kronecker theory the equivalent steps form the most delicate part of the proof. For the present, then, I am discontented, to say the least, when I find a geometric proof which does not explain in detail the projection mentioned. The transformation from the original plane curve to the non-singular space curve is relatively easy and can be done in a number of ways.

9. Origin of Theorem II. You will understand, I think, from what has been said above, that it is not easy to designate with certainty the origin of Theorem II. Halphen in 1884 ascribed the theorem to Noether, and he has since been imitated by a number of other writers, probably on account of the close relationship between Theorem II and Noether's Theorem I. On the basis of published papers it would be difficult to establish this origin. Klein, after stating the theorem without proof in the text of his Riemannsche Flächen, * inquires in a footnote "Wo ist der Satz zum ersten Male gedruckt?", and states that he himself first heard it orally from Kronecker in the autumn of 1869. Hensel and Landsberg also ascribe the theorem to Kronecker. If we rely on printed memoirs, however, one must admit that Kronecker's paper, first published in 1881, was devoted to the analysis of the discriminant of an algebraic function and was essentially analytic in character. His

* 1892, p. 245. 
reduction has indeed an important bearing upon Theorem II, but it affords only a partial proof. You will remember that Klein, in the footnote which he added to Bertini's Annalen paper of 1894, states that the geometric transformation there described was communicated to him orally by Clebsch in 1869 . Brill and Noether* further publish an extract from a letter of Klein to Noether dated December 17, 1869, in which Klein describes two results attained, respectively, by Kronecker and Clebsch. The first is the transformation of an arbitrary algebraic plane curve into another with no singularities except a single ordinary singular point; and the second is the transformation of a curve with a single ordinary singular point into one with at most ordinary double points by the geometric process which Klein ascribes to Clebsch in his footnote to Bertini's paper. Klein further adds in his letter that he believes Clebsch's method can be extended so as to reduce any number of arbitrary singularities to ordinary double points. Brill and Noether contend, and it seems to me with justice, that these assertions would be more effective as the basis of a claim for priority in the proof of the theorem if they had been afterwards substantiated in print. In 1897 Segre, $†$ speaking of the transformation of a plane curve into one in higher space with no singularities, says that solutions of the problem are contained implicitly in a paper by Brill and Noether of $1874, \ddagger$ and more explicitly in Veronese's paper of 1881 previously cited. As I said above, however, the first explicit published statement of the theorem with proof was probably that of Halphen in 1884. On the whole, I am inclined to agree with the conclusion implied by an above quoted statement of Bertini in 1891. The theorem seems to have gradually established itself in the consciousness of mathematicians during the decades from 1860 on, and an assignment of it to a definite origin would probably be incorrect. It is without doubt true that many a theorem now confidently attributed to a particular

* Jahresbericht, loc. cit., p. 371.

$\dagger$ Loc. cit., p. 43.

$\ddagger$ Mathematische Annalen, vol. 7 (1874), p. 269. 
mathematician would upon closer examination prove rather to be, as this one seems to be, the product of a gradual evolution in the minds of many individuals.

It seems to me that there is still a need for concise and clear proofs of the function-theoretic and the geometric Theorems II. The Hensel and Landsberg proof of the former, and Walker's proof of the latter following Bertini and Klein, are the only published ones with whose details I am at the present time personally well content. There are objections to both of them. It would be very difficult and inconvenient to analyze and understand the former unless one had read many of the preceding pages of Hensel and Landsberg's book, and possibly also Kronecker's original memoir. Walker's proof has the sort of geometric background which should enable one to decide definitely whether or not all essential details have been thought of and suitably discussed. But it is very long, and, having been published privately, it has not been subjected to the close scrutiny of mathematicians in various centers which seems to me desirable. The last objection applies also to my own proof of which I spoke above. So far as I know there is at present only one person who has definitely, though somewhat timidly, expressed his approval of this effort of mine, and his identity I must leave you to surmise. In conclusion I have only one further hope to express, namely, that no new proof will appear in the very condensed style which makes it almost impossible to decide whether the theorem has really been established or not. There are a number of these already, and the accumulation of others would only add to the difficulties of every earnest searcher for the truth in this domain. It seems to me that brevity at the expense of clearness, in a situation of this sort, should not be mistaken for simplicity.

The University of Chicago 